\title{
Voriconazole and posaconazole therapeutic drug monitoring: a retrospective study
}

Whitley M. Yi ${ }^{1}$, Kelly E. Schoeppler ${ }^{2}$, Jaclyn Jaeger ${ }^{1}$, Scott W. Mueller ${ }^{3}$, Robert MacLaren ${ }^{3}$, Douglas N. Fish ${ }^{3}$ and Tyree H. Kiser ${ }^{3,4^{*}}$

\begin{abstract}
Background: Therapeutic drug monitoring (TDM) aims to minimize the clinical impact of posaconazole and voriconazole pharmacokinetic variability. However, its benefits on clinical outcomes are still being defined. Additionally, TDM data are limited for posaconazole IV and delayed-release tablet formulations among specific patient populations, including critically ill. The aim of this study was to determine the percentage of therapeutic posaconazole and voriconazole drug levels across all formulations in a real-world clinical setting and elucidate factors affecting attainment of target concentrations.
\end{abstract}

Methods: This study was a retrospective cohort study conducted at the University of Colorado Hospital between September 2006 and June 2015 that evaluated patients who received posaconazole or voriconazole TDM as part of routine care.

Results: Voriconazole $(n=250)$ and posaconazole $(n=100)$ levels were analyzed from 151 patients. Of these, $54 \%$ of voriconazole and $69 \%$ of posaconazole levels were therapeutic. For posaconazole, 14/38 (37\%), 28/29 (97\%) and 27/33 (82\%) levels were therapeutic for the oral suspension, IV, and delayed-release tablet, respectively. Intravenous and delayed-release tablet posaconazole were 20 fold $(p<0.01)$ and sevenfold $(p=0.002)$ more likely than the oral suspension to achieve a therapeutic level. Subsequent levels were more likely to be therapeutic after dose adjustments (OR 3.31; 95\% Cl 1.3-8.6; $p=0.02$ ), regardless of timing of initial non-therapeutic level. In a multivariable logistic regression analysis, no characteristics were independently predictive of therapeutic voriconazole levels and only absence of H2RA/PPI use was independently predictive of therapeutic posaconazole levels. There was no correlation between survival and therapeutic drug levels for either voriconazole $(p=0.67)$ or posaconazole $(p=0.50)$.

Conclusions: A high percentage of drug levels did not achieve TDM targets for voriconazole and posaconazole oral suspension, supporting the need for routine TDM for those formulations. The utility of TDM for the IV and delayedrelease tablet formulations of posaconazole is less apparent.

Keywords: Voriconazole, Posaconazole, Therapeutic drug monitoring, Invasive fungal disease

\section{Background}

Posaconazole and voriconazole are triazoles with broad spectrum antifungal activity; however, their pharmacokinetics pose a therapeutic challenge. Voriconazole is used

\footnotetext{
*Correspondence: Ty.Kiser@ucdenver.edu

${ }^{4}$ Department of Clinical Pharmacy, University of Colorado Anschutz Medical Campus, 12850 E Montview Blvd, C238, Aurora, CO 80045, USA Full list of author information is available at the end of the article
}

first line for invasive aspergillosis (IA), while posaconazole is indicated as salvage therapy and prophylaxis for IA [1] and has activity against Mucorales [2]. Both antifungals exhibit highly variable inter- and intra-patient pharmacokinetics [3, 4], prompting development of newer dosage forms of posaconazole aimed at minimizing pharmacokinetic variability. Numerous factors have been associated with variability in voriconazole and posaconazole plasma levels; examples include altered 
intestinal absorption, drug interactions, diarrhea, chemotherapy, age, and weight [5-9].

For both voriconazole and posaconazole, the relationship between efficacy and drug exposure has been established [7, 10, 11]. A voriconazole plasma level of $1.0-5.5$ or $1.0-6.0 \mu \mathrm{g} / \mathrm{ml}$ is generally recommended as the goal range for improved outcomes and minimized toxicities [11, 12], and a threshold of $>0.5-0.7 \mu \mathrm{g} / \mathrm{ml}$ has been recommended as a therapeutic posaconazole concentration [13, 14]. Given high risk of mortality for invasive fungal disease and the inter- and intra-patient variability in posaconazole and voriconazole pharmacokinetics, therapeutic drug monitoring (TDM) is a strategy employed to optimize drug therapy. There has been an increase in TDM practices for antifungal agents [15], especially with voriconazole [16]. Additionally, the Infectious Diseases Society of America (IDSA) guidelines for aspergillosis recommend steadystate TDM for azole-based treatment of invasive aspergillosis [1]. However, as noted in the IDSA guidelines, more studies are needed to look at the benefit of TDM specifically for the intravenous and delayed-release tablet formulations of posaconazole, which has not been well-established.

A predominant focus of voriconazole and posaconazole TDM studies has been on hematologic cancer patient populations. Few studies have assessed other patient populations, such as the critically ill, who may have the lowest rates of attaining therapeutic drug levels of posaconazole [17]. TDM practices can be different in the intensive care unit (ICU) as opposed to other less emergent settings, as seen with the high rate of drug levels drawn on day 1 or 2 of therapy [18]. While guidelines recommend waiting until steady state for first TDM measurement [1], studies have not evaluated whether this is strictly adhered to in routine practice and what the implications on efficacy are if it is not.

This study took a broad look at patients receiving TDM for voriconazole and posaconazole, including patients in the critical care setting. The aim of this study was to determine the percentage of therapeutic posaconazole and voriconazole drug levels across all available formulations in a real-world clinical setting, and elucidate patient characteristics that affect ability to attain target plasma drug concentrations.

\section{Methods}

\section{Study design}

This study was a retrospective cohort study conducted at the University of Colorado Hospital between September 2006 and June 2015 . The study was approved by the Colorado Multiple Institutional Review Board.

\section{Patients}

Patients were identified through the University of Colorado Hospital's Electronic Health Record. Patients were included in the study if they had received voriconazole or posaconazole and had a drug level drawn for TDM as part of routine clinical care. Exclusion criteria included patients older than 89 or younger than 18 years, patients who were incarcerated, or patients who were pregnant.

\section{Drug levels}

All drug levels were drawn as part of routine care, with the decision to perform TDM based on the care team. Voriconazole trough drug levels were designated as therapeutic if they were $>1$ and $<5.5 \mu \mathrm{g} / \mathrm{ml}$. Posaconazole trough drug levels were designated as therapeutic if they were $>0.7 \mu \mathrm{g} / \mathrm{ml}$ [14]. All drug levels were evaluated via HPLC analysis at the Clinical Laboratory Improvement Amendments (CLIA) certified National Jewish Clinical Pharmacology lab (Denver, Colorado). The medical team received all results in real time for dose adjustments to be made if needed.

For analysis, all drug levels were treated as individual data points unless indicated otherwise. For voriconazole and posaconazole, steady state was defined as day 5 of therapy or later, whether after the initiation of therapy or a change in dose. When performing analyses between patients, rather than individual drug levels, patients were categorized as therapeutic or not based on the mean of their drug concentration measurements, as reported in previous TDM studies $[10,19]$. If a patient had alternated between therapy on voriconazole and posaconazole, the mean of each drug was evaluated separately, and these patients were included in both the voriconazole and posaconazole treatment groups for analysis.

\section{Interacting medications}

For voriconazole, interacting medications included amiodarone, diltiazem, erythromycin, phenobarbital, phenytoin, rifampin, and valproic acid. Posaconazole was considered to have the same potential interactions with the addition of metoclopramide.

\section{Invasive fungal disease assessment}

Invasive fungal disease (IFD) was classified as possible, probable, or proven per the European Organization for Research and Treatment of Cancer/Invasive Fungal Infections Cooperative Group and the National Institute of Allergy and Infectious Diseases Mycoses Study Group (EORTC/MSG) Consensus Group [20] with the following modifications: if a patient met mycological criteria and clinical criteria, the infection was classified as probable, even in the absence of a host factor. This was to account 
for immunocompromising patient factors outside those listed by EORTC/MSG as host factors. Patients who came in with a diagnosed IFD from an outside hospital were classified as probable. Patients with criteria for a proven infection from a previous hospital admission were classified as proven if still undergoing active treatment for the same infection. Immunocompromised status was defined as neutropenia, allogeneic stem cell transplant, solid organ transplant, prolonged use of corticosteroids, or T-cell immunosuppressive therapy.

Treatment failure was defined as discontinuation due to an adverse event, a breakthrough infection during prophylaxis, continued positive cultures, or worsening or persistent positive imaging for proven or probable IFD. Patient follow-up for clinical outcomes was assessed only up until patient discharge. Mortality was determined based on patient survival to hospital discharge. If patients had more than one hospital admission, the most recent admission was used in categorizing survival or treatment failure.

\section{Primary outcome}

The primary outcome evaluated the percentage of voriconazole and posaconazole plasma trough levels within goal.

\section{Secondary outcomes}

The secondary outcomes for the study were the percentage of patients unable to achieve a mean therapeutic voriconazole or posaconazole plasma concentration; correlation of clinical outcomes and adverse events with patient mean plasma concentration; correlation of concomitant $\mathrm{H} 2$ receptor antagonist/proton pump inhibitor (H2RA/PPI) use and dosage formulation with achievement of therapeutic plasma levels; comparison of the percentage of therapeutic posaconazole and voriconazole levels achieved among specific patient populations; percentage of posaconazole and voriconazole TDM measurements that were drawn prior to steady-state; the percentage of therapeutic drug levels achieved after dose modification; and the correlation between pre and post steady-state plasma concentrations without a dose adjustment.

\section{Statistical analysis}

Categorical data were compared via the Fisher exact test. Continuous data were evaluated using the Wilcoxon rank sum or the Student $t$-test, depending on data distribution. A matched pair analysis was used to evaluate the relationship between levels pre and post steady-state. Multivariable logistic regression was utilized to evaluate the impact of patient characteristics and drug dosages on achieving therapeutic drug concentrations. Variables with a $\mathrm{p}$ value $<0.1$ in the univariate analysis were included in the model (weight, TDD, $\mathrm{mg} / \mathrm{kg} / \mathrm{dose}, \mathrm{mg} / \mathrm{kg} /$ day, immunosuppression, ICU, and concomitant H2RA/PPI use). JMP Pro version 12.0.1 (SAS, Cary, North Carolina) was utilized to perform all analyses. A $p$ value $<0.05$ was considered significant.

\section{Results \\ Study population}

A total of 350 drug levels were analyzed from 151 patients. Of the 151 patients, 79 (48\%) were male, 93 (62\%) were Caucasian, 68 (45\%) were critically ill, and 61 $(40 \%)$ were transplant recipients (Table 1). Median age was 50 years (IQR 33-60 years) (Table 1). For patients with identified fungal pathogens, Aspergillus (37 of 103; 36\%) was the most common, followed by Candida (34 of 103; 33\%) (Table 1). There were 122 patients who received voriconazole TDM and 38 who received posaconazole TDM. Nine patients were counted in both drug groups, as they had sequentially taken voriconazole and posaconazole and had TDM performed on both.

\section{Drug levels}

Of the 350 drug levels, 250 voriconazole and 100 posaconazole trough levels were analyzed. Figure 1 displays voriconazole drug concentration by TDD ( $\mathrm{mg} /$ day) and weight-normalized TDD $(\mathrm{mg} / \mathrm{kg} /$ day). The median voriconazole plasma concentration was $2.43 \mu \mathrm{g} / \mathrm{ml}$ (Table 2) and the median posaconazole plasma concentration was $1.26 \mu \mathrm{g} / \mathrm{ml}$ (Table 3). Figure 2 displays posaconazole drug concentration by TDD (mg/day) and weight-normalized TDD $(\mathrm{mg} / \mathrm{kg} /$ day) and type of formulation. For voriconazole, 134 of 250 (54\%) levels were in the therapeutic range. Of the 116 non-therapeutic voriconazole levels, 65 were subtherapeutic and 51 were supratherapeutic. For posaconazole, 69 of $100(69 \%)$ levels were $>0.7 \mu \mathrm{g} /$ $\mathrm{ml}$. The mean number of drug levels drawn per patient was 2.3 levels. For patients taking voriconazole, 74 (61\%) patients maintained an average drug level that was in the therapeutic range, and for posaconazole, $22(58 \%)$ patients maintained an average drug level that was therapeutic (Table 1).

Overall, 126 (36\%) levels were drawn prior to steady state. For voriconazole, 96 (38\%) levels were pre-steady state and $30(30 \%)$ for posaconazole. Of these early levels, 42 (43\%) (19 subtherapeutic; 23 supratherapeutic) and 12 (40\%) were non-therapeutic for voriconazole and posaconazole, respectively. The mean \pm SD in-hospital length of therapy among those who were discharged on an antifungal or did not survive to discharge was $20.0 \pm 18.0$ and $18.6 \pm 18.5$ days for voriconazole and posaconazole, respectively. 
Table 1 Patient characteristics

\begin{tabular}{|c|c|c|c|}
\hline Characteristics & Overall $(n=151)$ & Voriconazole $(n=122)$ & Posaconazole $(n=38)$ \\
\hline Male & $79(48)$ & $60(49)$ & $22(58)$ \\
\hline Age (year) (median [IQR]) & $50(33-60)$ & $50(33-60)$ & $48(33-60)$ \\
\hline Weight ${ }^{\mathrm{a}}(\mathrm{kg})$ (median [IQR]) & $70.8(59.8-82.6)$ & $69.7(58.6-82.1)$ & $74.0(63.4-84.0)^{b}$ \\
\hline \multicolumn{4}{|l|}{ Race and ethnicity } \\
\hline Caucasian & $93(62)$ & $77(63)$ & $21(55)$ \\
\hline African American & $10(7)$ & $7(6)$ & $3(8)$ \\
\hline American India or Alaskan native & $3(2)$ & $3(2)$ & $1(3)$ \\
\hline Asian & $3(2)$ & $3(2)$ & 0 \\
\hline Hispanic & $19(12)$ & $12(10)$ & $8(21)$ \\
\hline Unknown & $23(15)$ & $20(17)$ & $5(13)$ \\
\hline Critically ill & $68(45)$ & $57(47)$ & $16(42)$ \\
\hline \multicolumn{4}{|l|}{ Transplant status } \\
\hline Solid organ recipients & $23(15)$ & $18(15)$ & $7(18)$ \\
\hline Bone marrow recipients & $38(25)$ & $33(27)$ & $10(26)$ \\
\hline \multicolumn{4}{|l|}{ Comorbidities } \\
\hline Cystic fibrosis & $18(12)$ & $17(14)$ & $1(3)$ \\
\hline \multicolumn{4}{|l|}{ Type of infection } \\
\hline Prophylaxis & $9(6)$ & $5(4)$ & $4(11)$ \\
\hline Possible & $32(21)$ & $27(22)$ & $10(26)$ \\
\hline Probable & $57(38)$ & $51(42)$ & $6(16)$ \\
\hline Proven & $53(35)$ & $39(32)$ & $18(47)$ \\
\hline Identified organism ( $n=103)$ & & $(n=82)$ & $(n=21)$ \\
\hline Candida spp. & & $31(38)$ & $3(14)$ \\
\hline Aspergillus spp. & & $34(41)$ & $3(14)$ \\
\hline Mucor & & 0 & $11(52)$ \\
\hline Scedosporium spp. & & $7(9)$ & 0 \\
\hline Cryptococcus & & $1(1)$ & $1(4)$ \\
\hline Other & & $9(11)$ & $3(14)$ \\
\hline \multicolumn{4}{|l|}{ Outcomes } \\
\hline Achieved a mean therapeutic level & & $74(61)$ & $22(58)$ \\
\hline Survived to hospital discharge & $109(72)$ & $90(74)$ & $26(68)$ \\
\hline Treatment failure & $39(26)$ & $33(27)$ & $7(18)$ \\
\hline
\end{tabular}

Data are presented as $\mathrm{n}(\%)$ or median (interquartile range)

$I Q R$ interquartile range

a Mean weight for each individual was used if there was $>1$ value

b $\mathrm{n}=37$ (weight for one individual unavailable)

Pharmacokinetic parameters and patient characteristics When comparing therapeutic versus non-therapeutic levels of combined voriconazole and posaconazole, type of organism was statistically different between groups $(p<0.01)$, while the only patient characteristic significantly different between groups was race/ ethnicity $(p<0.01)$ (Table 4$)$. When analyzing voriconazole and posaconazole levels individually, no patient characteristics were significantly correlated with achieving a therapeutic drug level for voriconazole. However, among non-therapeutic posaconazole drug levels, immunocompromised status $(p=0.04)$ and bone marrow transplant recipient status $(p=0.08)$ were more common (Table 3). Concomitant H2RA or PPI use was present with 28 of 38 (74\%) posaconazole oral suspension levels and 19 of 33 (58\%) posaconazole delayed-release tablet levels (Table 5). Both formulations had a higher proportion of concomitant H2RA/ PPI use among the non-therapeutic drug level group compared to the proportion in the therapeutic drug level group (Table 5). The odds ratio of achieving a therapeutic level with posaconazole suspension and concomitant H2RA/PPI use was 0.07 (95\% CI $0.01-$ $0.41 ; p<0.01)$. 


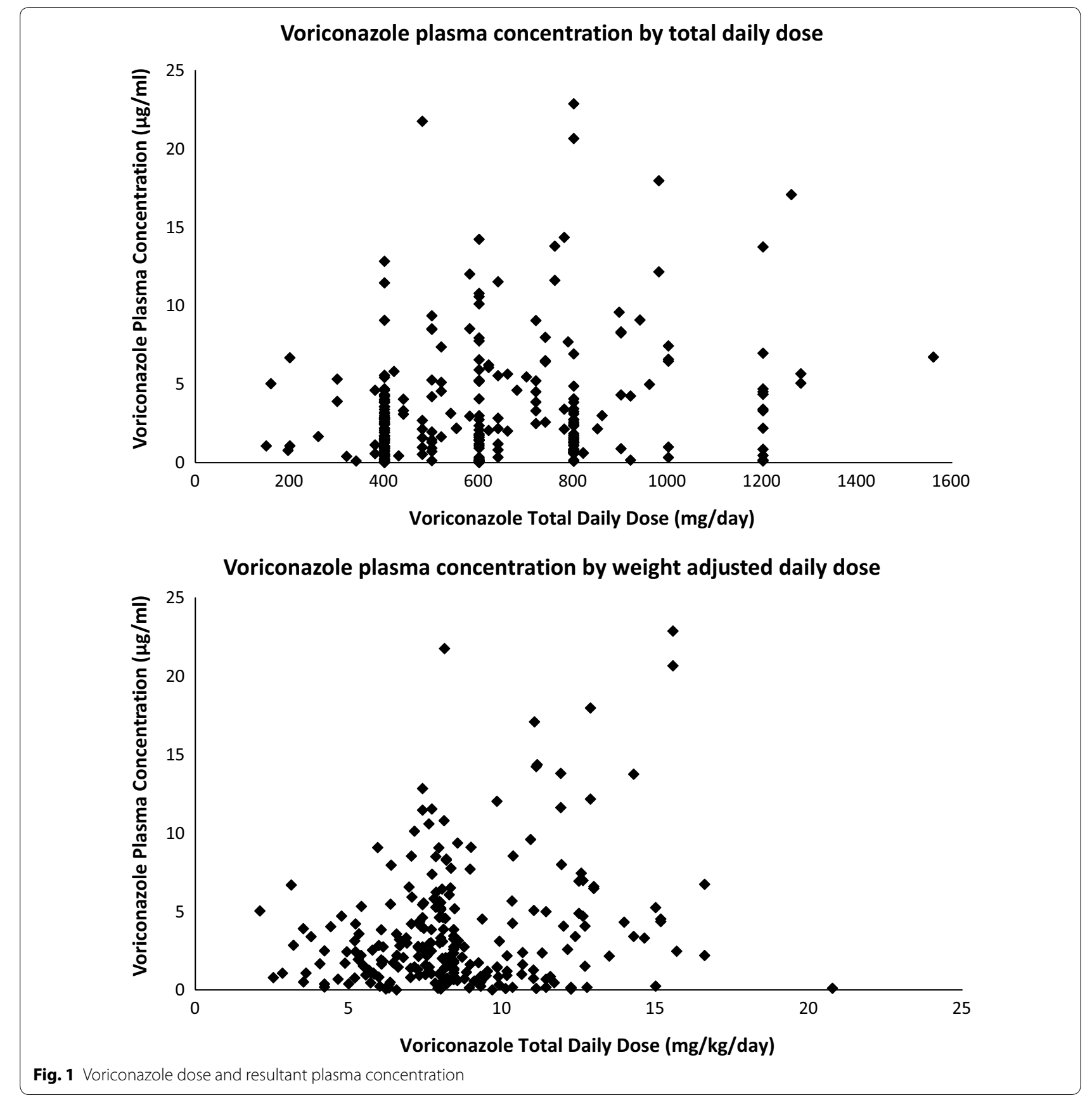

Patient weight, total daily dose (TDD), and weightnormalized TDD (in $\mathrm{mg} / \mathrm{kg} /$ day) were significantly correlated with achievement of goal posaconazole drug level (Table 3). However, when looking just at initial levels, only weight-normalized TDD (in $\mathrm{mg} / \mathrm{kg} /$ day) was significantly correlated with achievement of goal posaconazole level. For voriconazole, weight-normalized TDD (in mg/ $\mathrm{kg} /$ day), $\mathrm{mg} / \mathrm{kg} /$ dose, and TDD were significantly correlated with achievement of voriconazole goal drug level, regardless of looking at all levels or just initial levels (Table 2). Weight was not significantly correlated with voriconazole therapeutic drug levels. This was true for both subtherapeutic and supratherapeutic voriconazole levels (Table 2).

In a multivariable logistic regression analysis, using factors shown to be statistically significant through univariate analysis, only concomitant H2RA/PPI use was an independent determinant of a non-therapeutic 
Table 2 Voriconazole

\begin{tabular}{|c|c|c|c|}
\hline \multicolumn{4}{|l|}{ Overall $(n=250)$} \\
\hline \multicolumn{2}{|l|}{ Drug concentration $(\mu \mathrm{g} / \mathrm{ml})$ (median [IQR]) } & \multicolumn{2}{|l|}{$2.43(0.94-4.90)$} \\
\hline Comparison by drug level & $\begin{array}{l}\text { Therapeutic drug } \\
\text { level }(n=134)\end{array}$ & $\begin{array}{l}\text { Non-therapeutic drug } \\
\text { level }(n=116)\end{array}$ & $p$ \\
\hline \multicolumn{4}{|l|}{ PK parameters } \\
\hline Drug concentration ( $\mu \mathrm{g} / \mathrm{ml})$ (median [IQR]) & $2.6(1.66-3.87)$ & $0.90(0.45-7.97)$ & 0.10 \\
\hline Total daily dose (mg) (median [IQR]) & $552(400-800)$ & $600(405-800)$ & 0.01 \\
\hline mg/kg/dose (median [IQR]) & $3.95(3.3-4.4)$ & $4.24(3.7-5.5)$ & 0.01 \\
\hline mg/kg/day (median [IQR]) & $7.76(6.4-8.7)$ & $8.48(7.5-11.0)$ & $<0.01$ \\
\hline Weight (median [IQR]) & $70.8(61.0-84.3)$ & $75.5(58.6-88.75)$ & 0.65 \\
\hline Subtherapeutic $(n=65)$ & & $72(56.5-94)$ & 0.90 \\
\hline Supratherapeutic $(n=51)$ & & $86.1(76.1-102.7)$ & 0.36 \\
\hline Concomitant interacting medications & $13(10)$ & $15(13)$ & 0.43 \\
\hline Concomitant H2RA/PPI & $113(85)$ & $92(79)$ & 0.25 \\
\hline PK parameters_-initial dose only & $(n=66)$ & $(n=57)$ & \\
\hline mg/kg/dose (median [IQR]) & $3.67(2.9-4.2)$ & $4.03(3.6-4.7)$ & 0.01 \\
\hline mg/kg/day (median [IQR]) & $7.34(5.8-8.4)$ & $8.07(7.1-9.3)$ & 0.01 \\
\hline Weight (median [IQR]) & $69.0(60.5-82)^{\mathrm{a}}$ & $74.0(58.1-85.5)$ & 0.69 \\
\hline Subtherapeutic $(n=29)$ & & $64.4(49.5-79.3)$ & 0.11 \\
\hline Supratherapeutic $(n=28)$ & & $80.5(68.1-88.8)$ & 0.054 \\
\hline \multicolumn{4}{|l|}{ Patient characteristics } \\
\hline Critically ill & $54(40)$ & $47(41)$ & 1.00 \\
\hline Solid organ transplant recipients & $15(11)$ & $17(15)$ & 0.45 \\
\hline Bone marrow transplant recipients & $41(31)$ & $28(24)$ & 0.26 \\
\hline Cystic fibrosis & $17(13)$ & $11(9)$ & 0.55 \\
\hline Immunocompromised & $90(67)$ & $81(70)$ & 0.68 \\
\hline \multicolumn{4}{|l|}{ Outcomes } \\
\hline Length of stay (days) (median [IQR]) & $31(14-56)$ & $27(8-41)$ & 0.12 \\
\hline Adverse drug event requiring discontinuation & $1(0.7)$ & $8(7)$ & 0.01 \\
\hline Treatment failure & $35(26)$ & $44(38)$ & 0.06 \\
\hline Comparison by patient & $\begin{array}{l}\text { Therapeutic mean drug } \\
\text { level }(n=74)\end{array}$ & $\begin{array}{l}\text { Non-therapeutic mean drug } \\
\text { level }(n=48)\end{array}$ & \\
\hline \multicolumn{4}{|l|}{ Outcomes } \\
\hline Survive to hospital discharge $(n=122)$ & $56(76)$ & $34(71)$ & 0.67 \\
\hline Treatment failure & $12(16)$ & $21(44)$ & $<0.01$ \\
\hline Length of stay (days) (median [IQR]) & $22(11-37)$ & $21(7-37)$ & 0.25 \\
\hline
\end{tabular}

Data are presented as $\mathrm{n}$ (\%) or median (interquartile range)

$I Q R$ interquartile range, $P K$ pharmacokinetic

${ }^{\mathrm{a}} \mathrm{n}=67$

posaconazole drug level $(p=0.04)$ (Table 6). Analyzing the same parameters, no factors were shown to be independent determinants for achievement of a therapeutic voriconazole drug level (Table 6).

\section{Efficacy and adverse events}

Achieving target mean drug concentration did not significantly affect survival to hospital discharge for either antifungal, however, there was a $37 \%$ reduction in length of hospital stay for the therapeutic posaconazole level group $(p<0.01)$ (Table 3). This relationship remained significant when accounting for patients who did not survive to hospital discharge $(p<0.01)$. Additionally, there was a $42 \%$ reduction in treatment failure for patients able to maintain a therapeutic mean voriconazole drug level $(p<0.01)$ (Table 2). Overall, nine voriconazole levels were associated with an adverse drug event (ADE) that led to drug discontinuation. The reported ADEs were hallucinations $(\mathrm{n}=4)$, elevated liver enzymes $(\mathrm{n}=2)$, hyponatremia $(\mathrm{n}=1)$, renal insufficiency and altered mental 
Table 3 Posaconazole

\begin{tabular}{|c|c|c|c|}
\hline \multicolumn{4}{|l|}{ Overall $(n=100)$} \\
\hline \multicolumn{2}{|l|}{ Drug concentration ( $\mu \mathrm{g} / \mathrm{ml}$ ) (Median [IQR]) } & \multicolumn{2}{|l|}{$1.26(0.50-1.91)$} \\
\hline Comparison & $\begin{array}{l}\text { Therapeutic drug } \\
\text { level }(n=69)\end{array}$ & $\begin{array}{l}\text { Non-therapeutic drug } \\
\text { level }(n=31)\end{array}$ & $p$ \\
\hline \multicolumn{4}{|l|}{ PK parameters } \\
\hline Drug concentration ( $\mu \mathrm{g} / \mathrm{ml})$ (median [IQR]) & $1.61(1.17-2.30)$ & $0.19(0.15-0.48)$ & $<0.01$ \\
\hline Total daily dose (mg) (median [IQR]) & $300(300-400)$ & $800(600-1200)$ & $<0.01$ \\
\hline mg/kg/dose (median [IQR]) & $4.03(3.3-4.9)$ & $4.08(2.5-5.5)$ & 0.75 \\
\hline mg/kg/day (median [IQR]) & $4.68(3.8-7.5)$ & $9.67(5.5-13.9)$ & $<0.01$ \\
\hline Weight (median [IQR]) & $72(55.3-80.0)$ & $77(64.0-88.6)$ & 0.05 \\
\hline Concomitant interacting medication & $13(19)$ & $8(26)$ & 0.44 \\
\hline PK parameters_initial dose only & $(n=21)$ & $(n=17)$ & \\
\hline mg/kg/dose (median [IQR]) & $4.16(3.6-5.2)$ & $4.38(2.5-5.4)$ & 0.54 \\
\hline mg/kg/day (median [IQR]) & $5.67(4.0-9.6)$ & $10.49(5.4-14.4)$ & 0.04 \\
\hline Weight (median [IQR]) & $74(59.0-84.5)$ & $77(64.0-87.7)$ & 0.36 \\
\hline \multicolumn{4}{|l|}{ Patient characteristics } \\
\hline Critically ill & $34(49)$ & $16(52)$ & 1.00 \\
\hline Solid organ transplant recipients & $17(25)$ & $4(13)$ & 0.29 \\
\hline Bone marrow transplant recipients & $14(20)$ & $12(39)$ & 0.08 \\
\hline Cystic fibrosis & $1(1)$ & $1(3)$ & 0.53 \\
\hline Immunocompromised ${ }^{\mathrm{a}}$ & $41(59)$ & $25(81)$ & 0.04 \\
\hline \multicolumn{4}{|l|}{ Outcomes } \\
\hline Length of stay (days) (median [IQR]) & $27(17-48)$ & $43(36-76)$ & $<0.01$ \\
\hline Treatment failure & $13(18)$ & $9(30)$ & 0.30 \\
\hline Comparison by patient & $\begin{array}{l}\text { Therapeutic mean drug } \\
\text { level }(n=22)\end{array}$ & $\begin{array}{l}\text { Non-therapeutic mean drug } \\
\text { level }(n=16)\end{array}$ & \\
\hline \multicolumn{4}{|l|}{ Outcomes } \\
\hline Survive to hospital discharge $(n=38)$ & $14(64)$ & $12(75)$ & 0.50 \\
\hline Treatment failure & $4(18)$ & $3(18)$ & 1.00 \\
\hline Length of stay (days) (median [IQR]) & $23(8-36)$ & $40(35-57)$ & $<0.01$ \\
\hline
\end{tabular}

Data are presented as $\mathrm{n}(\%)$ or median (interquartile range)

$I Q R$ interquartile range

a Neutropenia, allogeneic stem cell transplant, solid organ transplant, prolonged use of corticosteroids, or T-cell immunosuppressive therapy

status $(\mathrm{n}=1)$, and intolerance $(\mathrm{n}=1)$. Non-therapeutic drug levels were greater than 9 times more likely to result in an $\mathrm{ADE}$ requiring drug discontinuation $(p=0.01)$ (Table 2). There were only three ADEs reported for posaconazole, all in the therapeutic drug group, with only one leading to discontinuation.

\section{Drug formulation}

Voriconazole dosage formulation was not significantly correlated to achieving therapeutic drug levels $(p=0.90)$ (Table 7). On the other hand, there was a statistically significant difference between posaconazole dosage formulation and achieving therapeutic drug level $(p<0.01)$. For the oral suspension, 14 of 38 levels (37\%) were therapeutic, while 28 of $29(94 \%)$ and 27 of $33(82 \%)$ were therapeutic for the intravenous and delayed release tablet, respectively (Table 7). When only comparing IV to PO posaconazole (all formulations), the IV formulation was 20 times more likely to have a therapeutic drug level (OR 20.5; 95\% CI 2.64-159; $p<0.01$ ). When specifically comparing the suspension to the delayed-release tablet, the delayed-release tablet was associated with a higher chance of achieving therapeutic levels (OR 7.7; 95\% CI 2.6-23.2; $p<0.01$ ).

\section{Dose adjustments}

There were 79 non-therapeutic drug levels in which the patient had a subsequent level measured. Only $41 \%$ of the time did the subsequent level reflect a dose adjustment. Dose adjustments to non-therapeutic levels 

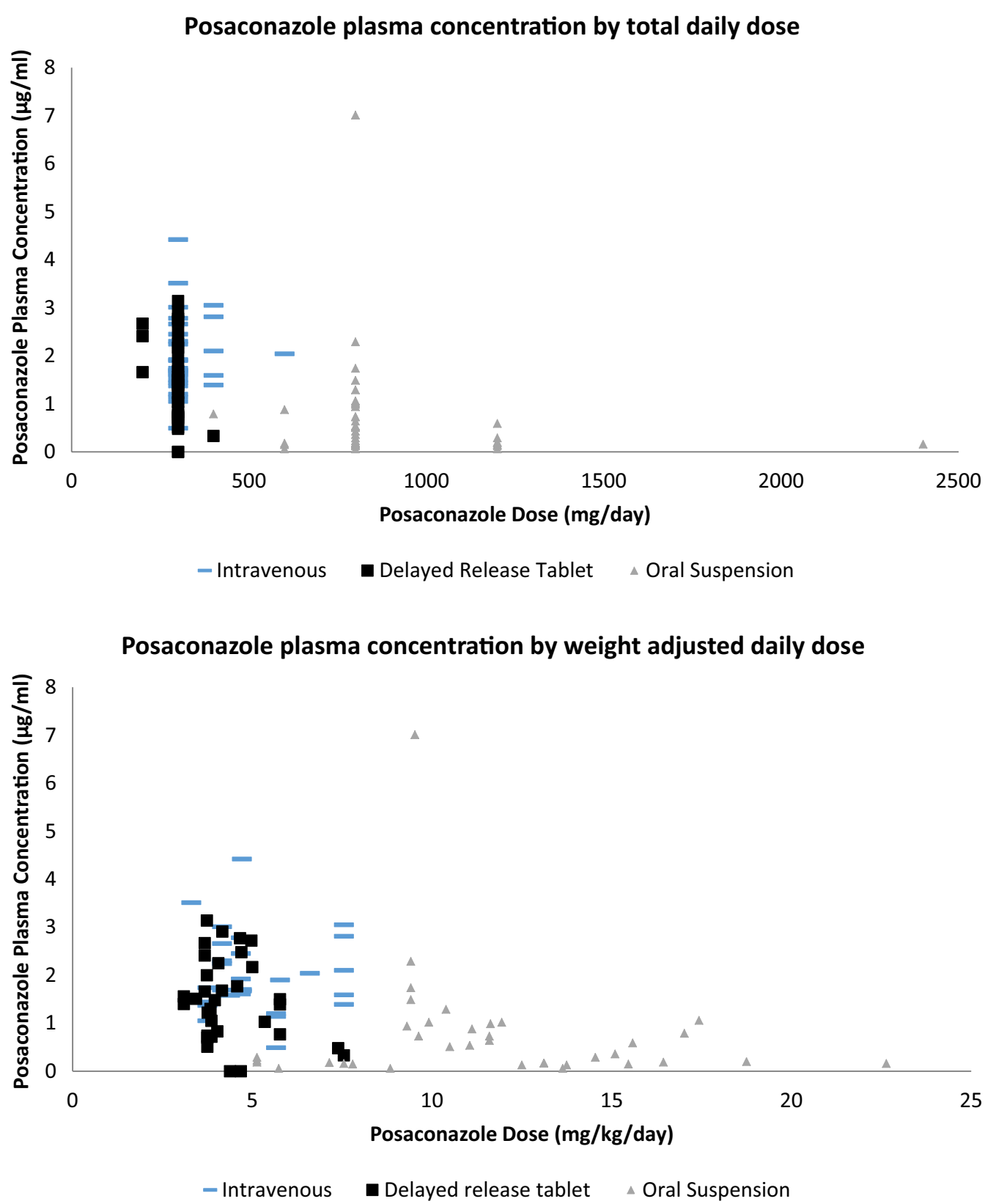

Fig. 2 Posaconazole dose and resultant plasma concentration

were associated with a statistically significant increase in achieving a subsequent therapeutic drug level (OR 3.31 ; $95 \%$ CI $1.3-8.6 ; p=0.02$ ), but did not correlate to increased survival to hospital discharge $(p=1.00)$ (Table 8). Of the 79 non-therapeutic levels, 32 were drawn prior to steady-state. When looking at these levels alone, dose adjustments were not statistically correlated with achievement of a subsequent therapeutic level $(p=0.68)$. However, the relationship was statistically significant when the initial non-therapeutic drug level was $\geq 3$ days into therapy ( $p=0.049$ ) (Table 8 ).

\section{Timing of drug levels}

In a matched pair analysis, there were 29 pairs of levels (voriconazole $=20$; posaconazole $=9$ ) with the first level drawn prior to steady state and the second level at steady state in which no dose adjustment was made. The pre-steady state concentrations were predictive of steady 
Table 4 Drug level comparison

\begin{tabular}{|c|c|c|c|}
\hline & $\begin{array}{l}\text { Therapeutic drug } \\
\text { level }(n=203)\end{array}$ & $\begin{array}{l}\text { Non-therapeutic drug } \\
\text { level }(n=147)\end{array}$ & $p$ \\
\hline \multicolumn{4}{|c|}{ Antifungal medication } \\
\hline Voriconazole & $134(66)$ & $116(79)$ & \multirow[t]{2}{*}{$<0.01$} \\
\hline Posaconazole & $69(34)$ & $31(21)$ & \\
\hline \multicolumn{4}{|l|}{ Timing of level } \\
\hline Pre-steady-state & $72(35)$ & $54(37)$ & \multirow[t]{2}{*}{0.82} \\
\hline Steady-state & $131(65)$ & $93(63)$ & \\
\hline Male & $98(48)$ & $84(57)$ & 0.11 \\
\hline $\begin{array}{l}\text { Weight (kg) } \\
\text { (median [IQR]) }\end{array}$ & $71.0(60.3-83.0)$ & $76.2(59.1-88.3)^{\mathrm{a}}$ & 0.13 \\
\hline $\begin{array}{l}\text { Age (year) } \\
\text { (median [IQR]) }\end{array}$ & $49(36-61)$ & $47(33-60)$ & 0.15 \\
\hline \multicolumn{3}{|l|}{ Race and ethnicity } & \multirow[t]{7}{*}{$<0.01$} \\
\hline Caucasian & $135(67)$ & $89(61)$ & \\
\hline African American & $20(10)$ & $6(4)$ & \\
\hline $\begin{array}{l}\text { American India } \\
\text { or Alaskan } \\
\text { native }\end{array}$ & $4(2)$ & $6(4)$ & \\
\hline Asian & $6(3)$ & 0 & \\
\hline Hispanic & $22(11)$ & $17(12)$ & \\
\hline Unknown & $16(8)$ & $29(20)$ & \\
\hline Critically III & $88(43)$ & $63(43)$ & 1.00 \\
\hline \multicolumn{4}{|l|}{ Transplant status } \\
\hline $\begin{array}{l}\text { Solid organ } \\
\text { recipients }\end{array}$ & $32(16)$ & $21(14)$ & 0.76 \\
\hline $\begin{array}{l}\text { Bone marrow } \\
\text { recipients }\end{array}$ & $55(27)$ & $40(27)$ & 1.00 \\
\hline \multicolumn{4}{|l|}{ Comorbidities } \\
\hline Cystic fibrosis & $18(9)$ & $12(8)$ & 0.85 \\
\hline Gastrointestinal & $46(23)$ & $25(17)$ & 0.23 \\
\hline \multicolumn{4}{|l|}{ Type of infection } \\
\hline Prophylaxis & $15(7)$ & $7(5)$ & \multirow[t]{4}{*}{0.38} \\
\hline Possible & $33(16)$ & $32(22)$ & \\
\hline Probable & $58(29)$ & $46(31)$ & \\
\hline Proven & $97(48)$ & $62(42)$ & \\
\hline \multicolumn{4}{|l|}{ Organism $(n=245)$} \\
\hline Candida spp. & $29(20)$ & $38(37)$ & \multirow[t]{6}{*}{$<0.01$} \\
\hline Aspergillus spp. & $48(33)$ & $34(34)$ & \\
\hline Mucor & $31(21)$ & $7(7)$ & \\
\hline $\begin{array}{l}\text { Scedosporium } \\
\text { spp. }\end{array}$ & $18(12)$ & $13(13)$ & \\
\hline Cryptococcus & $7(5)$ & 0 & \\
\hline Other & $13(9)$ & $9(9)$ & \\
\hline
\end{tabular}

Data are presented as $\mathrm{n}(\%)$ or median (interquartile range) IQR interquartile range

${ }^{a} \mathrm{n}=146$ (weight unavailable for one drug level)

state values, as there was no statistical difference between the pre and post steady state levels, with the mean difference in voriconazole levels being -0.509 (95\% CI $-1.21-$ $0.19 ; p=0.10)$ and the mean difference in posaconazole levels being -0.25 (95\% CI $-0.64-0.14 ; p=0.34)$.
Table 5 Posaconazole and concomitant PPI/H2RA use

\begin{tabular}{llll}
\hline $\begin{array}{l}\text { Concomitant use } \\
\text { of PPI or H2RA }\end{array}$ & $\begin{array}{l}\text { Therapeutic } \\
(\mathbf{n}=\mathbf{4 6})\end{array}$ & $\begin{array}{l}\text { Non-therapeutic } \\
(\mathbf{n}=\mathbf{2 9})\end{array}$ & $\boldsymbol{p}$ \\
\hline Intravenous & $(\mathrm{n}=28)$ & $(\mathrm{n}=1)$ & \\
With PPI/H2RA & $27(96)$ & $1(100)$ & 1.00 \\
No PPI/H2RA & $1(4)$ & 0 & \\
Oral Suspension & $(n=14)$ & $(n=24)$ & \\
With PPI/H2RA & $6(43)$ & $22(92)$ & \\
No PPI/H2RA & $8(57)$ & $2(8)$ & 0.01 \\
Delayed-release tablet & $(n=27)$ & $(n=6)$ & \\
With PPI/H2RA & $13(48)$ & $6(100)$ & \\
No PPI/H2RA & $14(52)$ & 0 & \\
\hline
\end{tabular}

Data are presented as $n(\%)$

Table 6 Variables independently associated with therapeutic drug levels

\begin{tabular}{|c|c|c|c|}
\hline Variable & $\begin{array}{l}\text { Multivariable logistic } \\
\text { regression estimate }\end{array}$ & $p$ & OR $(95 \% \mathrm{Cl})$ \\
\hline \multicolumn{4}{|l|}{ Voriconazole } \\
\hline Total daily dose (mg) & 0.001 & 0.64 & \\
\hline $\mathrm{mg} / \mathrm{kg} / \mathrm{dose}$ & -0.79 & 0.33 & \\
\hline $\mathrm{mg} / \mathrm{kg} /$ day & 0.44 & 0.29 & \\
\hline Weight & -0.006 & 0.78 & \\
\hline $\begin{array}{l}\text { Concomitant H2RA/ } \\
\text { PPI }\end{array}$ & 0.21 & 0.23 & $1.53(0.77-3.09)$ \\
\hline Critically ill & -0.06 & 0.67 & $0.88(0.51-1.53)$ \\
\hline $\begin{array}{l}\text { Immunocompro- } \\
\text { mised }\end{array}$ & 0.002 & 0.99 & $1.003(0.56-1.79)$ \\
\hline \multicolumn{4}{|l|}{ Posaconazole } \\
\hline Total daily dose (mg) & 0.009 & 0.07 & \\
\hline $\mathrm{mg} / \mathrm{kg} / \mathrm{dose}$ & -0.076 & 0.74 & \\
\hline $\mathrm{mg} / \mathrm{kg} /$ day & -0.16 & 0.62 & \\
\hline Weight & -0.015 & 0.65 & \\
\hline $\begin{array}{l}\text { Concomitant H2RA/ } \\
\text { PPI }\end{array}$ & -1.20 & 0.04 & $0.09(0.007-0.75)$ \\
\hline Critically ill & -0.34 & 0.43 & $0.05(0.07-2.45)$ \\
\hline $\begin{array}{l}\text { Immunocompro- } \\
\text { mised }\end{array}$ & -0.16 & 0.73 & $0.73(0.11-4.53)$ \\
\hline
\end{tabular}

$O R$ odds ratio, $\mathrm{Cl}$ confidence interval

\section{Discussion}

The main finding of the study was the high percentage of voriconazole and posaconazole levels not at goal. Fortysix percent of all voriconazole levels measured were outside the therapeutic range, and $39 \%$ of patients had an average voriconazole level also outside that range. Posaconazole had a lower percentage of total levels not at goal (31\%) but a higher percentage (42\%) of patients whose mean plasma level remained non-therapeutic. A possible explanation for the higher percentage of posaconazole 
Table 7 Comparison of dosage formulations

\begin{tabular}{|c|c|c|c|c|}
\hline \multicolumn{2}{|l|}{ Formulation } & \multicolumn{2}{|l|}{ Drug concentration $(\mu \mathrm{g} / \mathrm{ml})$} & $p$ \\
\hline \multicolumn{5}{|l|}{ Posaconazole } \\
\hline \multicolumn{2}{|c|}{ Delayed-release tablet (median [IQR]) } & \multicolumn{2}{|l|}{$1.48(0.76-2.21)$} & \multirow[t]{3}{*}{$<0.01$} \\
\hline \multicolumn{2}{|l|}{ IV (median [IQR]) } & \multicolumn{2}{|l|}{$1.74(1.47-2.56)$} & \\
\hline \multicolumn{2}{|c|}{ Oral suspension (median [IQR]) } & \multicolumn{2}{|l|}{$0.40(0.17-0.95)$} & \\
\hline \multicolumn{5}{|l|}{ Voriconazole } \\
\hline \multicolumn{2}{|l|}{ IV (median [IQR]) } & \multicolumn{2}{|l|}{$3.31(1.41-5.70)$} & $<0.01$ \\
\hline \multirow[t]{2}{*}{$\mathrm{PO}($ median $[\mathrm{IQR}])$} & & \multicolumn{2}{|l|}{$1.78(0.84-4.00)$} & \\
\hline & Therapeutic drug level & Non-therapeutic drug level & OR $(95 \% \mathrm{Cl})$ & $p$ \\
\hline Posaconazole & $(n=69)$ & $(n=31)$ & & \\
\hline Delayed-release tablet & $27 / 33(82 \%)$ & $6 / 33(18 \%)$ & $7.7(2.6-23.2)^{\mathrm{a}}$ & $<0.01$ \\
\hline IV & $28 / 29(97 \%)$ & $1 / 29(3 \%)$ & $48(5.9-392.3)^{\mathrm{a}}$ & $<0.01$ \\
\hline Oral suspension & $14 / 38(37 \%$ & 24/38 (63\%) & & \\
\hline Voriconazole & $(n=134)$ & $(n=116)$ & & \\
\hline IV (no. [\%]) & $56(42)$ & $50(43)$ & $1.06(0.64-1.74)$ & 0.90 \\
\hline $\mathrm{PO}$ (no. [\%]) & $78(58)$ & $66(57)$ & & \\
\hline
\end{tabular}

$I Q R$ interquartile range, $O R$ odds ratio, $\mathrm{Cl}$ confidence interval

a In reference to posaconazole oral suspension

Table 8 Analysis of patients with $\geq \mathbf{2}$ drug levels

\begin{tabular}{|c|c|c|c|c|}
\hline $\begin{array}{l}\text { Comparison action taken on an } \\
\text { initial non-therapeutic drug level }\end{array}$ & $\begin{array}{l}\text { Survive to hospital } \\
\text { discharge }(n=59)\end{array}$ & $\begin{array}{l}\text { Did not survive to hospital } \\
\text { discharge }(n=20)\end{array}$ & OR & $p$ \\
\hline A dose adjustment was made & $24(41)$ & $8(40)$ & $1.03(0.4-2.9)$ & 1.00 \\
\hline \multirow[t]{2}{*}{ No dose adjustment was made } & $35(59)$ & $12(60)$ & & \\
\hline & Therapeutic drug level & Non-therapeutic drug level & OR $(95 \% \mathrm{Cl})$ & $p$ \\
\hline Overall & $(n=29)$ & $(n=50)$ & & \\
\hline A dose adjustment was made (no. [\%]) & $17(59)$ & $15(30)$ & $3.31(1.3-8.6)$ & 0.02 \\
\hline No dose adjustment was made (no. [\%]) & $12(41)$ & $35(70)$ & & \\
\hline First level prior to steady state ${ }^{a}$ & $(n=9)$ & $(n=23)$ & & \\
\hline Dose adjustment made (no. [\%]) & $4(44)$ & $7(30)$ & $1.83(0.4-8.9)$ & 0.68 \\
\hline No dose adjustment made (no. [\%]) & $5(56)$ & $16(70)$ & & \\
\hline First level at steady state ${ }^{a}$ & $(n=20)$ & $(n=27)$ & & \\
\hline Dose adjustment made (no. [\%]) & $13(65)$ & $8(30)$ & $4.41(1.3-15.2)$ & 0.02 \\
\hline No dose adjustment made (no. [\%]) & $7(35)$ & $19(70)$ & & \\
\hline First level measured after $\geq 3$ days & $(n=28)$ & $(n=44)$ & & \\
\hline Dose adjustment made (no. [\%]) & $16(57)$ & $14(32)$ & $2.86(1.1-7.6)$ & 0.049 \\
\hline No dose adjustment made (no. [\%]) & $12(43)$ & $30(68)$ & & \\
\hline
\end{tabular}

$O R$ odds ratio, $\mathrm{Cl}$ confidence interval

a Stead state defined as $\geq 5$ days of therapy

patients unable to achieve a mean therapeutic level compared to voriconazole could be the lower rate of TDM in routine practice for posaconazole compared to voriconazole [15], which was a trend also seen at the site of this study. In addition to the potential inexperience in posaconazole TDM-based dose adjustments, the strategies needed for each formulation vary, and evidence has shown previously recommended dose adjustment strategies [14] to be less effective [21]. Even with optimizing posaconazole oral suspension by increasing frequency over dose, however, evidence has not been able to demonstrate a statistically significant difference between median plasma concentrations at baseline and after dose adjustments [21]. Overall, these data demonstrate the 
challenges of posaconazole TDM and appear to suggest a greater difficultly in successful posaconazole TDM compared to voriconazole TDM.

The $46 \%$ of non-therapeutic voriconazole levels was a little higher than reported in the majority of other studies, which ranged from 23 to $37 \%$ [9, 18, 22-25]. To our knowledge, only three studies reported percentages greater than $40 \%$ [26-28]. The large number of early levels in this study, however, may help explain the higher proportion of sub-therapeutic levels. When looking at posaconazole levels reported in other studies, the rate of not achieving optimal plasma concentrations varied widely from $20 \%$ [29] to $90 \%$ [30]; however, a majority of the studies report percentages in the range of $20-50 \%$ $[6,7,21,31-34]$. It can be difficult to compare percentages of therapeutic drug measurements across studies due to the differing therapeutic threshold cut-offs, TDM practices, and patient populations at each site. Overall though, it demonstrates that the increase in practicing routine TDM does not appear to translate yet to achievement of higher percentages of therapeutic drug levels.

While other studies have demonstrated correlations between drug level variation and patient characteristics [19], our study looked specifically at correlation between patient characteristics and ability to obtain a therapeutic drug level, not merely a statistical difference in levels. Surprisingly, many of the patient characteristics, such as cystic fibrosis and age $[9,35]$, that have been associated with drug level variations in either voriconazole or posaconazole in previous studies were not shown to significantly correlate with a therapeutic or non-therapeutic drug level in the current study. For some of the patient characteristics represented by a small sample size, such as cystic fibrosis, the potential for type II error should be considered. However, overall, the data suggests that the effects of patient characteristics on drug level might be more subtle than suggested and potentially less predictive in determining which patients might need preemptive dose adjustment or are more likely to not achieve goal plasma concentrations.

When evaluating patient characteristics for combined posaconazole and voriconazole, only race/ethnicity was significantly correlated with likelihood of achieving therapeutic levels. This is most likely driven by the known CYP2C19 pharmacogenomics variation in voriconazole metabolism [36]. When looking at posaconazole and voriconazole individually, weight normalized TDD was significantly correlated with achievement of therapeutic drug levels. Interestingly, for both antifungals, the nontherapeutic group had a higher median $\mathrm{mg} / \mathrm{kg} /$ day dose (Tables 2, 3). Possible explanations include serial dose increases for patients with persistently low plasma levels or the saturable absorption for voriconazole and the posaconazole oral suspension [21], evidenced by the low median drug level from posaconazole oral suspension compared to the other formulations in this study. Immunocompetence was significantly correlated with the ability to achieve therapeutic posaconazole levels, but not voriconazole. Another finding of this study was lack of weight being significantly correlated to ability to achieve initial therapeutic posaconazole drug levels. Previous evidence on weight and posaconazole has been conflicting. One study showed no correlation between weight and drug level with the delayed-release posaconazole tablet [37], while another study suggested that patients $\geq 90 \mathrm{~kg}$ had lower mean trough levels than those $<90 \mathrm{~kg}[6]$.

This is one of the first studies that has looked at all three dosage formulations of posaconazole. The analysis showed the dosage formulation is significantly correlated with ability to achieved a therapeutic plasma level, with the IV formulation having the highest percentage of therapeutic levels (Table 7). When only comparing the oral dosage forms, the delayed-release tablet was sevenfold more likely to achieve a therapeutic level. This is almost identical to the results found in another study comparing the suspension with the delayed-release tablet [37]. Although the delayed-release tablet seemed to predict greater success in achieving therapeutic levels, there are still limitations to its use in patients who are unable to swallow pills whole. Due to this, the critically ill patient population might have a lower percentage of patients able to take the delayed-release tablet. In addition, it can be cost-prohibitive. Several patients in this study switched back to the oral suspension at discharge due to cost.

With more consistent rates of therapeutic levels in this study, there appears to be less PK variability with the IV and delayed-release tablet formulations, indicating TDM might not have the same role for these formulations as for the oral suspension. Although, it is important to note that currently, only the oral suspension has been studied in efficacy trials for the treatment and prophylaxis of invasive aspergillosis [1].

Surprisingly, this study showed a significant correlation between PPI/H2RA use and therapeutic concentrations for both the posaconazole suspension and the delayedrelease tablet, while other studies have shown acid suppressing therapies not to significantly affect plasma levels of the delayed-release tablet $[37,38]$. Pham et al., however, correlated PPI/H2RA use with median plasma levels, while this study looked at correlation between PPI/ H2RA use and achievement of levels $>0.7 \mu \mathrm{g} / \mathrm{ml}$. This highlights some of the present difficulties in studying antifungal TDM and the ability to aggregate the data for clinical decision making.

This study had a large percentage of drug levels that were measured early, which could be a reflection of ICU 
practices, as nearly half of patients in this study were critically ill. A retrospective study of voriconazole in an ICU reported that $49 \%$ of the drug levels measured for TDM were too early [18]. Given the frequency of pre-steady state TDM in real-life ICU settings, we aimed to evaluate the utility of early drug measurements. In this study, the paired analysis showed no significant difference between voriconazole and posaconazole levels pre and post steady-state, indicating that early levels could be predicative of steady-state levels. However, the analysis reflects pooled posaconazole and voriconazole levels. These results must be interpreted in the context of other studies that have analyzed posaconazole and voriconazole individually. For example, data of early dose predictability showed a posaconazole plasma level of $\geq 0.338 \mu \mathrm{g} / \mathrm{ml}$ on day 3 predictive of a $0.5 \mu \mathrm{g} / \mathrm{ml}$ by day 7 [34]. While other studies have looked at early dose predictability, none of the studies to our knowledge looked at the effect of dose adjustments based on early drug levels. When analyzing dose adjustments, we found that dose adjustments made after a non-therapeutic level, regardless of the timing, tripled the chance of the subsequent level being in the therapeutic range (Table 8).

In this study, non-therapeutic voriconazole levels were associated with adverse drug events (ADEs) requiring discontinuation. Other studies have described how TDM can reduce the rate of voriconazole discontinuation due to ADEs [23, 39]. Unfortunately for this study, it was difficult to accurately assess the total number of adverse drug events requiring discontinuation due to the lack of ADEs reported in patient charts and inability to verify if an ADE was related to voriconazole. Overall, this study adds further support to the use of voriconazole TDM to help prevent ADEs leading to drug discontinuation.

This study did not demonstrate a correlation between survival and therapeutic drug level, which is a finding consistent with the majority of previous studies. Only a few studies have reported a correlation between voriconazole drug concentration and mortality [40-42]. Two of the studies are pediatric studies and the third reported a correlation between a first steady-state trough of voriconazole $\leq 0.35 \mathrm{mg} / \mathrm{l}$ and increased risk of mortality [42]. For posaconazole, it was not surprising to find a lack of correlation between survival and therapeutic drug level in this small sample size, given that a portion of the levels were for prophylaxis rather than treatment.

While many studies have shown a correlation between drug exposure and efficacy [13, 19, 34, 42], in this study neither drug showed a significant difference in treatment failure correlated with therapeutic drug levels. However, voriconazole did show a significant decrease in treatment failure when comparing average drug levels among patients (Table 2). Based on these data, mean drug level might be a more accurate endpoint to predict therapeutic failure. The treatment failure results of this study should be interpreted carefully though, due to the variable follow-up time in assessing if treatment failure had occurred. For many patients, treatment failure was unknown.

In looking at other clinical outcomes, posaconazole demonstrated a significant reduction in length of hospital stay for those who achieved therapeutic levels. Reduction in hospital stay reduces costs and is especially relevant for immunocompromised patients to minimize exposure to hospital acquired infections. Further pharmacoeconomic studies should be conducted to compare the costeffectiveness of each posaconazole formulation, given the large price variation between them.

This study has many limitations. As a retrospective study, some data, including treatment failure, were difficult to assess based on incomplete or sometimes missing information in patient charts. There was also a selection bias. Although TDM is routinely used for voriconazole and posaconazole at our institution, patients with the greatest concern for PK variability were more likely to receive TDM, resulting in a potentially falsely elevated non-therapeutic rate. With longer treatment durations for fungal infections, this study was also unable to assess the total length of voriconazole and posaconazole therapy, as data on therapy duration outside of the hospital stay was unavailable. Moreover, the study only assessed drug and dose at the time of the drug level measurement. Changes in the drug regimen in response to a non-therapeutic drug level were only seen if a second drug level was measured. Therefore, there were many potential changes to drug therapy that were not assessed in the study. Further, there could have been multiple changes to a patient's drug regimen between drug levels being drawn that might not be reflected in this study. The study also did not differentiate between treatment and prophylaxis goals for posaconazole. All posaconazole levels were considered therapeutic if $>0.7 \mu \mathrm{g} / \mathrm{ml}$. However, a trough of $>1 \mu \mathrm{g} / \mathrm{mL}$ is recommended for treatment in some guidelines [43].

While this study helps add to the evidence regarding TDM utility during posaconazole and voriconazole therapy, it does not address pharmacoeconomic considerations of TDM, evaluate efficacy between dose-adjustment strategies, or elucidate PK differences or TDM utility for treatment of targeted fungal infections. In order to create more comprehensive guidance for best TDM practices, these topics need to be addressed through prospective, randomized trials. In addition, large, prospective, randomized trials are needed to further assess the efficacy of voriconazole and posaconazole TDM on clinical outcomes, including mortality. Although studies suggest 
potential benefit in decreasing ADE-related drug discontinuation and treatment failure, most of the current studies fail to demonstrate clinical benefit in mortality with TDM.

\section{Conclusions}

In conclusion, the high percentage of non-therapeutic drug levels found in this study supports the benefit of TDM for optimization of drug levels following administration of voriconazole and posaconazole oral suspension in patients at high risk for mortality from fungal infections. The role of TDM for the intravenous and delayed release tablet formulations of posaconazole is less clear due to higher consistency in achievement of goal concentrations with these preparations. Additionally, there could be potential benefit in making dose adjustments from levels drawn prior to steady-state, though further studies are needed to validate these findings.

\begin{abstract}
Abbreviations
ADE: adverse drug event: CLIA: Clinical Laboratory Improvement Amendments; EORT/MSG: European Organization for Research and Treatment of Cancer/Invasive Fungal Infections Cooperative Group and the National Institute of Allergy and Infectious Diseases Mycoses Study Group; HPLC: high performance liquid chromatography; H2RA: histamine-2 receptor antagonist; IA: invasive aspergillosis; ICU: intensive care unit; IDSA: Infectious Diseases Society of America; IFD: invasive fungal disease; PPI: proton-pump inhibitor; TDM: therapeutic drug monitoring.
\end{abstract}

\section{Authors' contributions}

WMY participated in the study design, collected the data, performed statistical analyses and interpretation of the data, and drafted the manuscript. KES participated in the study design and data collection and critically revised the manuscript for important intellectual content. JJ assisted in data collection and critically revised the manuscript for important intellectual content. SWM participated in the study design and critically revised the manuscript for important intellectual content. RM participated in the study design and critically revised the manuscript for important intellectual content. DNF participated in the study design and critically revised the manuscript for important intellectual content. THK conceived the study, participated in its design, participated in analysis and interpretation of the data, helped draft the manuscript, and critically revised the manuscript for intellectual content. All authors read and approved the final manuscript.

\begin{abstract}
Author details
${ }^{1}$ University of Colorado, Skaggs School of Pharmacy and Pharmaceutical Sciences, 12850 E Montview Blvd, Aurora, CO 80045, USA. ${ }^{2}$ Department of Pharmacy, University of Colorado Hospital, 12605 E 16th Ave, Aurora, CO 80045, USA. ${ }^{3}$ Department of Clinical Pharmacy, University of Colorado, Skaggs School of Pharmacy and Pharmaceutical Sciences, 12850 E Montview Blvd, Aurora, CO 80045 , USA. ${ }^{4}$ Department of Clinical Pharmacy, University of Colorado Anschutz Medical Campus, 12850 E Montview Blvd, C238, Aurora, CO 80045, USA.
\end{abstract}

\section{Acknowledgements}

The authors thank Larry Stupke, Senior database administrator at the University of Colorado Health System, for his assistance with this project.

\section{Competing interests}

The authors declare that they have no competing interests.

\section{Availability of data and materials}

The datasets used and/or analyzed during the current study are available from the corresponding author on reasonable request.
Consent for publication

Not applicable.

Ethics approval and consent to participate

This study was approved by the Colorado Multiple Institutional Review Board (Protocol 11-1227).

\section{Funding}

The study was self-funded.

\section{Publisher's Note}

Springer Nature remains neutral with regard to jurisdictional claims in published maps and institutional affiliations.

Received: 7 March 2017 Accepted: 31 August 2017

Published online: 11 September 2017

\section{References}

1. Patterson TF, Thompson GR 3rd, Denning DW, Fishman JA, Hadley S, Herbrecht $\mathrm{R}$, et al. Practice guidelines for the diagnosis and management of aspergillosis: 2016 update by the Infectious Diseases Society of America. Clin Infect Dis. 2016;63(4):e1-60.

2. Groll AH, Walsh TJ. Posaconazole: clinical pharmacology and potential for management of fungal infections. Expert Rev Anti Infect Ther. 2005;3(4):467-87.

3. Dekkers BG, Bakker M, van der Elst KC, Sturkenboom MG, Veringa A, Span LF, Alffenaar JC. Therapeutic drug monitoring of posaconazole: an update. Curr Fungal Infect Rep. 2016;10:51-61.

4. Wang $T$, Xie J, Wang Y, Zheng $X$, Lei J, Wang $X$, et al. Pharmacokinetic and pharmacodynamic properties of oral voriconazole in patients with invasive fungal infections. Pharmacotherapy. 2015;35(9):797-804.

5. Andes D. Optimizing antifungal choice and administration. Curr Med Res Opin. 2013;29(Suppl 4):13-8.

6. Miceli MH, Perissinotti AJ, Kauffman CA, Couriel DR. Serum posaconazole levels among haematological cancer patients taking extended release tablets is affected by body weight and diarrhoea: single centre retrospective analysis. Mycoses. 2015;58(7):432-6.

7. van der Elst KC, Brouwers $\mathrm{CH}$, van den Heuvel ER, van Wanrooy MJ, Uges DR, van der Werf TS, Kosterink JG, Span LF, Alffenaar JW. Subtherapeutic posaconazole exposure and treatment outcome in patients with invasive fungal disease. Ther Drug Monit. 2015;37(6):766-71.

8. Vehreschild JJ, Muller C, Farowski F, Vehreschild MJ, Cornely OA, Fuhr U, Kreuzer KA, Hallek M, Kohl V. Factors influencing the pharmacokinetics of prophylactic posaconazole oral suspension in patients with acute myeloid leukemia or myelodysplastic syndrome. Eur J Clin Pharmacol. 2012;68(6):987-95.

9. Cabral-Galeano E, Ruiz-Camps I, Len-Abad O, Pou-Clave L, Sorde-Masip R, Meije-Castillo Y, et al. Clinical usefulness of therapeutic drug monitoring of voriconazole in a university hospital. Enferm Infecc Microbiol Clin. 2015;33(5):298-302.

10. Troke PF, Hockey HP, Hope WW. Observational study of the clinical efficacy of voriconazole and its relationship to plasma concentrations in patients. Antimicrob Agents Chemother. 2011;55(10):4782-8.

11. Pascual A, Calandra T, Bolay S, Buclin T, Bille J, Marchetti O. Voriconazole therapeutic drug monitoring in patients with invasive mycoses improves efficacy and safety outcomes. Clin Infect Dis. 2008;46(2):201-11.

12. Luong ML, Al-Dabbagh M, Groll AH, Racil Z, Nannya Y, Mitsani D, Husain S. Utility of voriconazole therapeutic drug monitoring: a meta-analysis. J Antimicrob Chemother. 2016;71(7):1786-99.

13. Dolton MJ, Ray JE, Chen SC, Ng K, Pont L, McLachlan AJ. Multicenter study of posaconazole therapeutic drug monitoring: exposure-response relationship and factors affecting concentration. Antimicrob Agents Chemother. 2012;56(11):5503-10.

14. Jang SH, Colangelo PM, Gobburu JV. Exposure-response of posaconazole used for prophylaxis against invasive fungal infections: evaluating the need to adjust doses based on drug concentrations in plasma. Clin Pharmacol Ther. 2010;88(1):115-9. 
15. Neoh CF, Snell Gl, Kotsimbos T, Levvey B, Morrissey CO, Slavin MA, Stewart $\mathrm{K}$, Kong DC. Antifungal prophylaxis in lung transplantation-a worldwide survey. Am J Transpl. 2011;11(2):361-6.

16. Wiederhold NP, Pennick GJ, Dorsey SA, Furmaga W, Lewis JS 2nd, Patterson TF, Sutton DA, Fothergill AW. A reference laboratory experience of clinically achievable voriconazole, posaconazole, and itraconazole concentrations within the bloodstream and cerebral spinal fluid. Antimicrob Agents Chemother. 2014;58(1):424-31.

17. Ray J, Campbell L, Rudham S, Nguyen Q, Marriott D. Posaconazole plasma concentrations in critically ill patients. Ther Drug Monit. 2011;33(4):387-92.

18. van Wanrooy MJ, Rodgers MG, Span LF, Zijlstra JG, Uges DR, Kosterink JG, van der WerfTS, Alffenaar JW. Voriconazole therapeutic drug monitoring practices in intensive care. Ther Drug Monit. 2016;38(3):313-8.

19. Dolton MJ, Ray JE, Chen SC, Ng K, Pont LG, McLachlan AJ. Multicenter study of voriconazole pharmacokinetics and therapeutic drug monitoring. Antimicrob Agents Chemother. 2012;56(9):4793-9.

20. De Pauw B, Walsh TJ, Donnelly JP, Stevens DA, Edwards JE, Calandra T, et al. Revised definitions of invasive fungal disease from the European organization for research and treatment of cancer/invasive fungal infections cooperative group and the national institute of allergy and infectious diseases mycoses study group (EORTC/MSG) consensus group. Clin Infect Dis. 2008;46(12):1813-21.

21. Hummert SE, Green MR. Therapeutic drug monitoring and dose adjustment of posaconazole oral suspension in adults with acute myeloid leukemia. Ther Drug Monit. 2015;37(4):508-11.

22. Guinea J, Escribano P, Marcos-Zambrano LJ, Pelaez T, Kestler M, Munoz P, Vena A, Lopez-Fabal F, Bouza E. Therapeutic drug monitoring of voriconazole helps to decrease the percentage of patients with off-target trough serum levels. Med Mycol. 2016;54(4):353-60.

23. Park WB, Kim NH, Kim KH, Lee SH, Nam WS, Yoon SH, et al. The effect of therapeutic drug monitoring on safety and efficacy of voriconazole in invasive fungal infections: a randomized controlled trial. Clin Infect Dis. 2012;55(8):1080-7.

24. Franz CE, Lyons MJ, O'Brien R, Panizzon MS, Kim K, Bhat R, et al. A 35-year longitudinal assessment of cognition and midlife depression symptoms: the Vietnam Era Twin Study of Aging. Am J Geriatr Psychiatry. 2011;19(6):559-70.

25. Kim SH, Lee DG, Kwon JC, Lee HJ, Cho SY, Park C, et al. Clinical impact of cytochromeP450 2C19 genotype on the treatment of invasive aspergillosis under routine therapeutic drug monitoring of voriconazole in a Korean population. Infect Chemother. 2013;45(4):406-14.

26. Chu HY, Jain R, Xie H, Pottinger P, Fredricks DN. Voriconazole therapeutic drug monitoring: retrospective cohort study of the relationship to clinical outcomes and adverse events. BMC Infect Dis. 2013;13:105.

27. Miyakis S, van Hal SJ, Solvag CJ, Ray J, Marriott D. Clinician ordering practices for voriconazole therapeutic drug monitoring: experiences of a referral laboratory. Ther Drug Monit. 2010;32(5):661-4.

28. Mitsani D, Nguyen MH, Shields RK, Toyoda Y, Kwak EJ, Silveira FP, et al. Prospective, observational study of voriconazole therapeutic drug monitoring among lung transplant recipients receiving prophylaxis: factors impacting levels of and associations between serum troughs, efficacy, and toxicity. Antimicrob Agents Chemother. 2012;56(5):2371-7.

29. Park WB, Cho JY, Park SI, Kim EJ, Yoon S, Yoon SH, et al. Effectiveness of increasing the frequency of posaconazole syrup administration to achieve optimal plasma concentrations in patients with haematological malignancy. Int J Antimicrob Agents. 2016;48(1):106-10.
30. Bryant AM, Slain D, Cumpston A, Craig M. A post-marketing evaluation of posaconazole plasma concentrations in neutropenic patients with haematological malignancy receiving posaconazole prophylaxis. Int J Antimicrob Agents. 2011;37(3):266-9.

31. Lebeaux D, Lanternier F, Elie C, Suarez F, Buzyn A, Viard JP, et al. Therapeutic drug monitoring of posaconazole: a monocentric study with 54 adults. Antimicrob Agents Chemother. 2009:53(12):5224-9.

32. Hoenigl M, Duettmann W, Raggam RB, Huber-Krassnitzer B, Theiler G, Seeber K, et al. Impact of structured personal on-site patient education on low posaconazole plasma concentrations in patients with haematological malignancies. Int J Antimicrob Agents. 2014;44(2):140-4.

33. Gross BN, lhorst $G$, Jung M, Wasch R, Engelhardt M. Posaconazole therapeutic drug monitoring in the real-life setting: a single-center experience and review of the literature. Pharmacotherapy. 2013;33(10):1117-25.

34. Chae H, Cho SY, Yu H, Cha K, Lee S, Kim M, et al. Determination of posaconazole concentration with LC-MS/MS in adult patients with hematologic malignancy. Clin Chim Acta. 2015;450:220-6.

35. Zhang H, Nguyen MH, Clancy CJ, Joshi R, Zhao W, Ensor C, Venkataramanan R, Shields RK. Pharmacokinetics of posaconazole suspension in lung transplant patients with and without cystic fibrosis. Antimicrob Agents Chemother. 2016;60(6):3558-62.

36. Lamoureux F, Duflot T, Woillard JB, Metsu D, Pereira T, Compagnon P, et al. Impact of CYP2C19 genetic polymorphisms on voriconazole dosing and exposure in adult patients with invasive fungal infections. Int J Antimicrob Agents. 2016;47(2):124-31.

37. Pham AN, Bubalo JS, Lewis JS 2nd. Comparison of posaconazole serum concentrations from haematological cancer patients on posaconazole tablet and oral suspension for treatment and prevention of invasive fungal infections. Mycoses. 2016;59:226.

38. Cumpston A, Caddell R, Shillingburg A, Lu X, Wen S, Hamadani M, Craig M, Kanate AS. Superior serum concentrations with posaconazole delayed-release tablets compared to suspension formulation in hematological malignancies. Antimicrob Agents Chemother. 2015;59(8):4424-8.

39. Matsumoto K, Abematsu K, Shigemi A, Kanazawa N, Watanabe E, Yokoyama Y, Ikawa K, Morikawa N, Takeda Y. Therapeutic drug monitoring of voriconazole in Japanese patients: analysis based on clinical practice data. J Chemother. 2016;28(3):198-202.

40. Neely M, Rushing T, Kovacs A, Jelliffe R, Hoffman J. Voriconazole pharmacokinetics and pharmacodynamics in children. Clin Infect Dis. 2010;50(1):27-36.

41. Soler-Palacin P, Frick MA, Martin-Nalda A, Lanaspa M, Pou L, Rosello E, de Heredia CD, Figueras C. Voriconazole drug monitoring in the management of invasive fungal infection in immunocompromised children: a prospective study. J Antimicrob Chemother. 2012:67(3):700-6.

42. Miyakis S, van Hal SJ, Ray J, Marriott D. Voriconazole concentrations and outcome of invasive fungal infections. Clin Microbiol Infect. 2010;16(7):927-33.

43. Chau MM, Kong DC, van Hal SJ, Urbancic K, Trubiano JA, Cassumbhoy $M$, et al. Consensus guidelines for optimising antifungal drug delivery and monitoring to avoid toxicity and improve outcomes in patients with haematological malignancy, 2014. Intern Med J. 2014;44(12b):1364-88.

\section{Submit your next manuscript to BioMed Central and we will help you at every step:}

- We accept pre-submission inquiries

- Our selector tool helps you to find the most relevant journal

- We provide round the clock customer support

- Convenient online submission

- Thorough peer review

- Inclusion in PubMed and all major indexing services

- Maximum visibility for your research

Submit your manuscript at www.biomedcentral.com/submit

C Biomed Central 